\title{
A Case of Grisel Syndrome Showing No Underlying Laxity of the Atlanto-axial Joint
}

\author{
Ah-Reum Ahn, MD, Yul-Hyun Park, MD, Eun Ji Park, MD, Shin-Young Yim, MD, PhD
}

The Center for Torticollis, Department of Physical Medicine and Rehabilitation, Ajou University School of Medicine, Suwon, Korea

\begin{abstract}
Grisel syndrome is a rare, non-traumatic atlanto-axial subluxation associated with an inflammatory or infectious process in the upper neck. According to the two-hit hypothesis, which is widely accepted for the pathogenesis of Grisel syndrome, preexisting ligamentous laxity of the atlanto-axial joint is regarded as the first hit. An inflammatory or infectious process of the atlanto-axial joint acts as the second hit, resulting in non-traumatic atlanto-axial subluxation. We report on a 6-year-old girl with atlanto-axial subluxation following retropharyngeal and cervical lymphadenitis. She was diagnosed with Grisel syndrome, for which an initial computed tomography did not show any preexisting ligamentous laxity of the atlanto-axial joint. A literature review found only 4 case reports on Grisel syndrome with an initially normal atlanto-axial joint. The present case offers some evidence that a single hit, such as inflammatory changes in the atlanto-axial joint, might cause Grisel syndrome, even without underlying ligamentous laxity.
\end{abstract}

Keywords Atlanto-axial joint, Torticollis, Joint instability

\section{INTRODUCTION}

Grisel syndrome is a rare, non-traumatic atlanto-axial subluxation associated with an inflammatory or infectious process of the upper neck. It is characterized by torticollis and a limited range of motion of the neck along with pain. Grisel syndrome is one of the differential diagnoses of patients with abnormal posture of the head and neck, and it mainly affects children after an upper respiratory tract infection or neck surgery, such as an adenoidectomy and tonsillectomy $[1,2]$. According to a search on MEDLINE as of May 2016, 105 cases have been reported since 1930, when Pierre Grisel described patients presenting torticollis and atlanto-axial subluxation following pharyngitis. The incidence of Grisel syndrome remains unknown, and 4 cases have been reported in Korea [3].

With an early diagnosis, patients with Grisel syndrome can recover with conservative treatment, but surgical treatment may be required when the diagnosis is delayed

Received June 23, 2016; Accepted August 24, 2016

Corresponding author: Shin-Young Yim

The Center for Torticollis, Department of Physical Medicine and Rehabilitation, Ajou University School of Medicine, 164 World cup-ro, Yeongtong-gu, Suwon 16499, Korea. Tel: +82-31-219-5284, Fax: +82-31-219-5209, E-mail: syyim@ajou.ac.kr

ORCID: Ah-Reum Ahn (http://orcid.org/0000-0002-5279-184X); Yul-Hyun Park (http://orcid.org/0000-0003-3103-293X); Eun Ji Park (http://orcid. org/0000-0002-4679-5553); Shin-Young Yim (http://orcid.org/0000-0002-8521-6331).

(c) This is an open-access article distributed under the terms of the Creative Commons Attribution Non-Commercial License (http://creativecommons.org/ licenses/by-nc/4.0) which permits unrestricted noncommercial use, distribution, and reproduction in any medium, provided the original work is properly cited. Copyright ( 2017 by Korean Academy of Rehabilitation Medicine 
[1]. Neurologic complications have been reported, with outcomes ranging from radiculopathy to tetraplegia and even death [4]. Therefore, early diagnosis and treatment is important to avoid serious sequelae.

Although the pathogenesis of Grisel syndrome remains unclear, the two-hit hypothesis proposed by Battiata and Pazos [5] in 2004 is the most widely accepted. This hypothesis states that preexisting ligamentous laxity of the atlanto-axial joint is the first hit in the pathogenesis of Grisel syndrome, and inflammatory mediators carried to the cervical muscles act as the second hit, resulting in muscle spasm and subsequent atlanto-axial subluxation. Another hypothesis is that an inflammatory or infectious process extends to the atlanto-axial joint through the pharyngo-vertebral veins via numerous lymphovenous anastomoses, resulting in a laxity of the atlanto-axial ligaments and/or bony erosion of the insertion site of the ligaments without preexisting ligamentous laxity of the
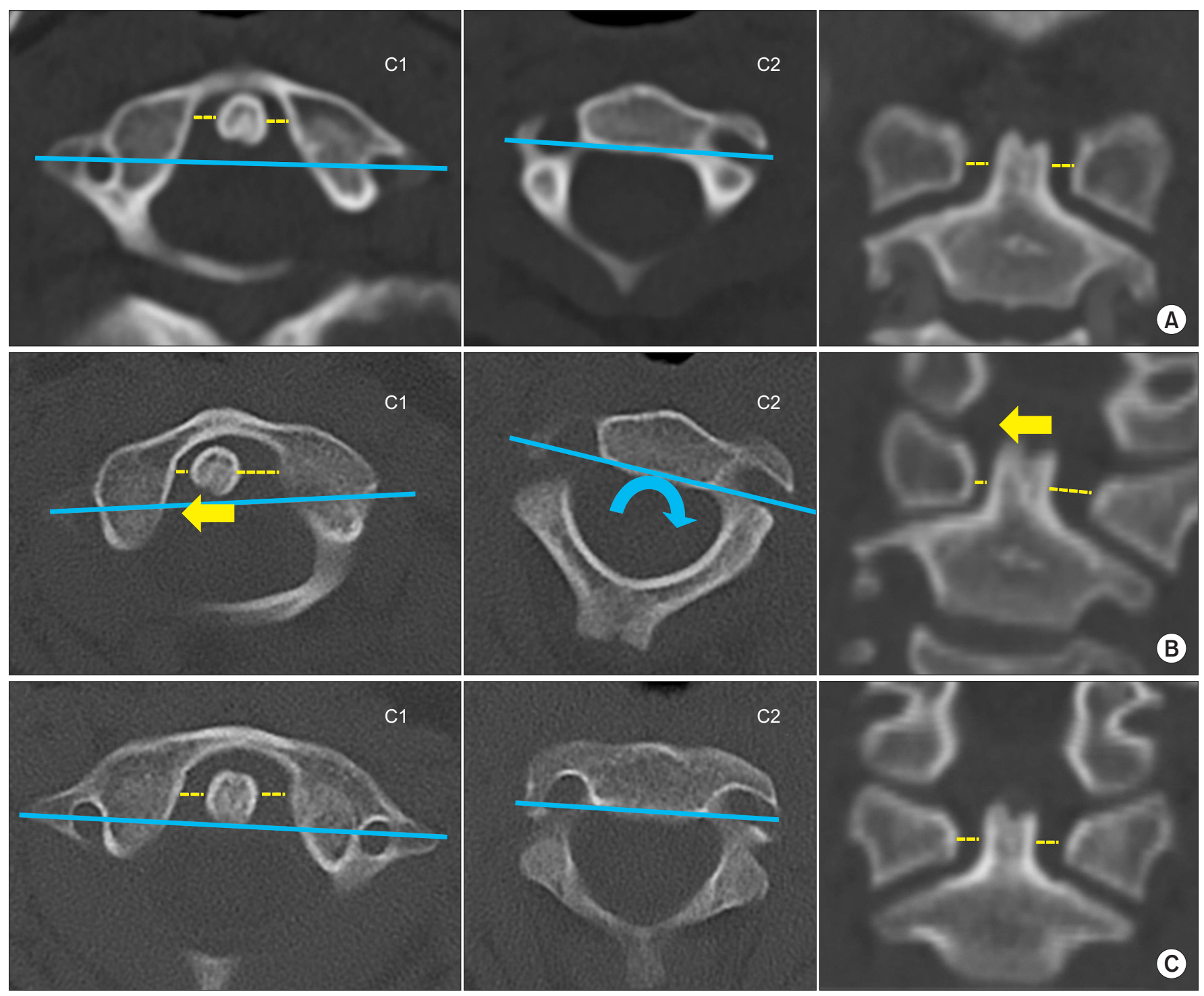

Fig. 1. Serial neck computed tomography (CT). (A) The initial CT on admission shows a normal alignment of the atlanto-axial joint with symmetric lateral atlanto-dens interval and without rotation of the atlanto-axial joint. (B) CT taken after the onset of torticollis shows rotation of the atlanto-axial joint (blue curved arrow) with a lateral shift of the dens toward the right (yellow arrow). (C) CT on the 28th hospital day shows a normal alignment of the atlanto-axial joint. $\mathrm{C} 1$, the atlas; $\mathrm{C} 2$, the axis; blue line, a line connecting bilateral transverse foramina of the atlas and the axis; yellow dotted line, the lateral atlanto-dens interval. 
atlanto-axial joint $[6,7]$. This might be considered a single-hit hypothesis in the current report, in contrast with the two-hit hypothesis.

A literature review of 105 reports on Grisel syndrome found only 4 case reports showing an initially normal atlanto-axial joint. We attended to a 6 -year-old girl with Grisel syndrome who did not show any preexisting ligamentous laxity of the atlanto-axial joint on initial computed tomography (CT). We present this case along with serial radiological images, suggesting that Grisel syndrome could be caused by a single hit, such as an inflammatory change in the atlanto-axial joint, even without underlying ligamentous laxity of the atlanto-axial joint.

\section{CASE REPORT}

A 6-year-old girl with swelling in her left neck and fever for 4 days prior was admitted to the Department of Pediatrics via the emergency room. She had no history of trauma of the head and neck.

Enlarged lymph nodes around the left sternocleidomastoid muscle were observed, and a neck CT with contrast on the day of admission showed fluid collection in the retropharyngeal space, along with swelling of the adenoids, palatine tonsils, and multiple retropharyngeal and left cervical lymph nodes. A CT showed a normal alignment of the atlanto-axial joint with an anterior atlanto-dens interval of $1.66 \mathrm{~mm}$, indicating no preexisting ligamentous laxity (Fig. 1A).

The day after admission, she developed abnormal head posture along with severe neck pain (Fig. 2). She was transferred to the Department of Physical Medicine and Rehabilitation for further evaluation and management. On physical examination, she showed severe limitation of the neck motion with only $10^{\circ}$ of neck rotation toward the left and $10^{\circ}$ of neck lateral flexion toward the right. There was no evidence of neurological impairment. Followup CT revealed type I atlanto-axial rotatory subluxation by the Fielding and Hawkins classification, with a lateral shift of the dens toward the right (Fig. 1B). Magnetic resonance imaging (MRI) of the neck showed abnormal enhancement of the atlanto-axial ligaments and the posterior longitudinal ligament at the level of the $\mathrm{C} 2$ vertebra, as well as fluid collection in the retropharyngeal space, indicating inflammatory changes in the retropharyngeal space and craniovertebral junction (Fig. 3).

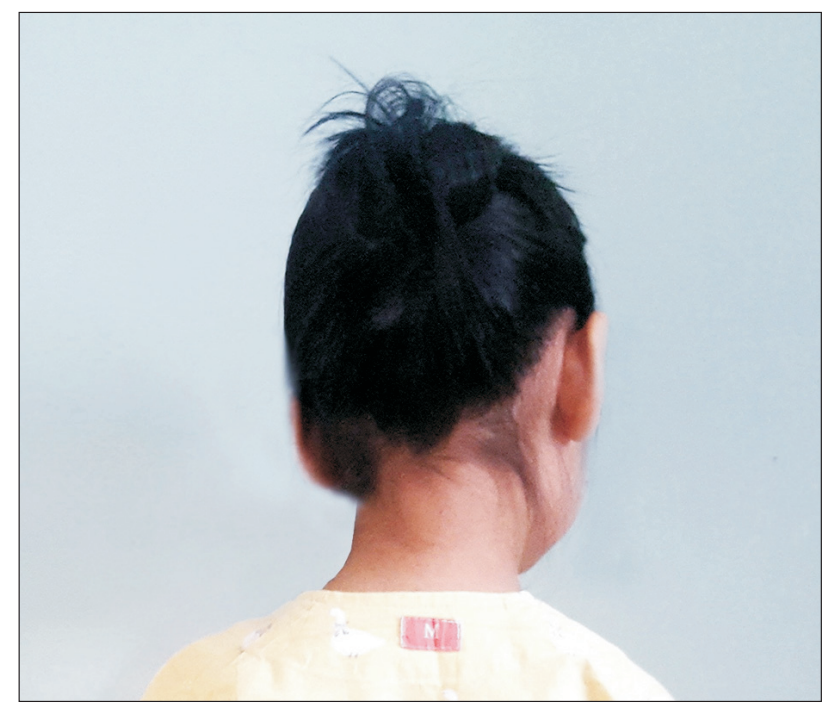

Fig. 2. A 6-year-old girl with Grisel syndrome showing abnormal head posture.

With a diagnosis of Grisel syndrome, continuous cervical halter traction and intermittent cervical traction were administered, along with non-steroidal anti-inflammatory drugs and muscle relaxants. Intermittent cervical traction was combined with continuous cervical traction for a more effective reduction of the subluxation from the 5 th day of treatment, when pain had decreased. Intermittent cervical traction was administered once a day for 20 minutes with a traction weight of 4.5 pounds $(2 \mathrm{~kg})$ that was gradually increased to 5 pounds $(2.3 \mathrm{~kg})$. On the 8th day of cervical traction, she showed full range of neck motion. A follow-up CT showed normal alignment of the atlanto-axial joint (Fig. 1C). She was discharged on the 28th hospital day and continued to wear a Philadelphia neck collar for 6 weeks after discharge. The symptom remission was sustained after discontinuing the use of the neck collar.

\section{DISCUSSION}

We presented a case with Grisel syndrome, where initial CT did not show any pre-existing ligamentous laxity of the atlanto-axial joint. A literature review found 4 case reports on Grisel syndrome with an initially normal atlanto-axial joint. A diagnosis of Grisel syndrome should be considered in patients with acute torticollis after infection or surgery of the head and neck, even in the absence of underlying conditions associated with a laxity of the 

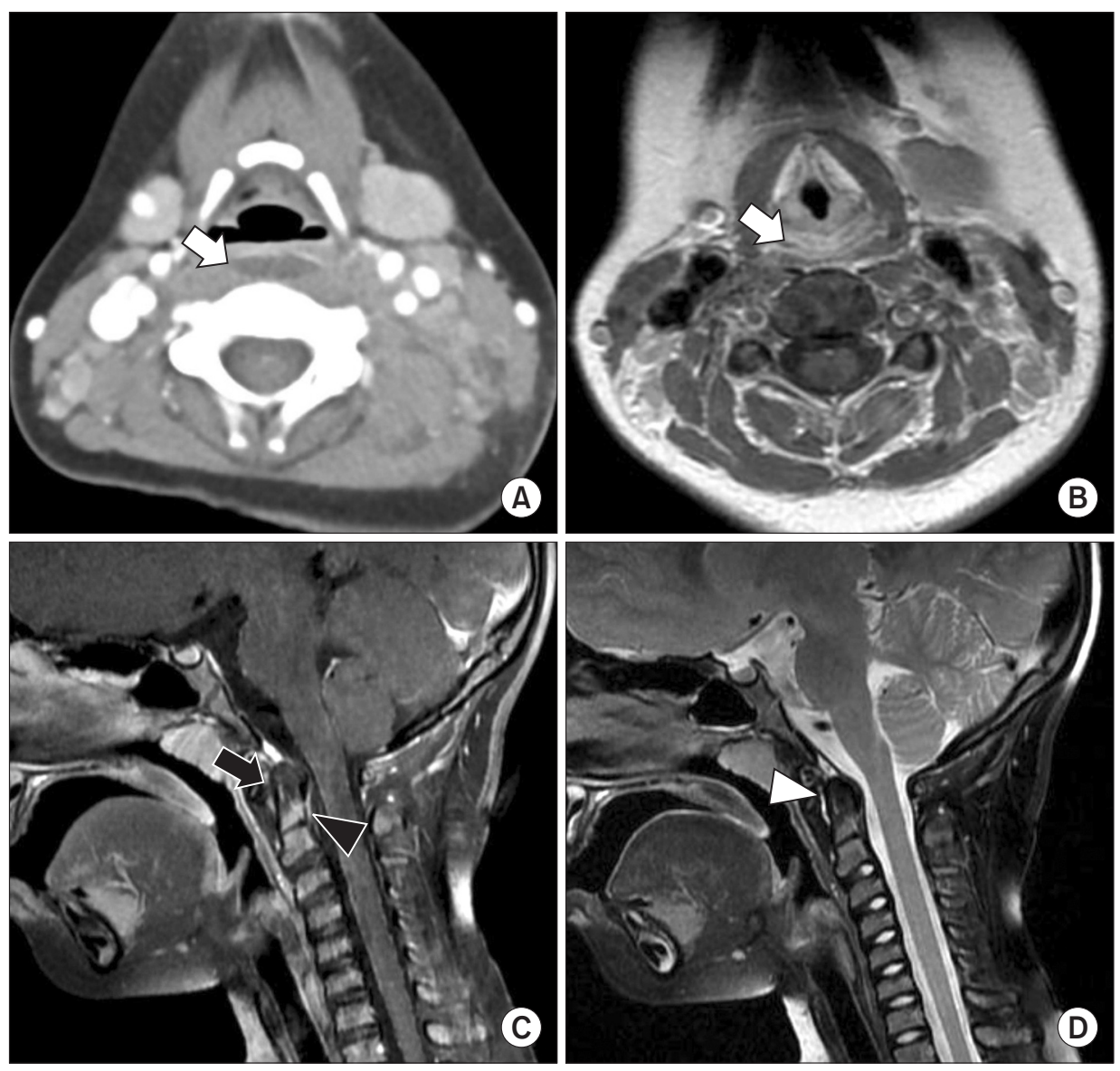

Fig. 3. Radiographic studies showing inflammatory changes of the soft tissue. (A) Computed tomography with contrast shows a diffuse, low attenuating lesion in the retropharyngeal space, indicating a fluid collection due to inflammation (white arrow). (B) Axial gadolinium-enhanced T1-weighted magnetic resonance imaging (MRI) shows a fluid collection in the retropharyngeal space (white arrow). (C) Sagittal gadoliniumenhanced T1-weighted MRI shows abnormal enhancement of ligaments of the atlanto-axial joint (black arrow) and posterior longitudinal ligament at the level of the C2 vertebra (black arrow head), suggesting inflammatory changes of the soft tissue. (D) Sagittal T2weighted MRI shows a fluid collection in the atlanto-axial joint space (white arrow head). atlanto-axial joint. In Korea, 4 cases of Grisel syndrome have been reported, following courses of acute cervical lymphadenitis, upper respiratory tract infection, pulmonary tuberculosis and tonsillectomy [3].

The pathogenesis of Grisel syndrome remains unclear. In 1984, Parke et al. [7] described the pharyngo-vertebral veins, a previously undescribed system of veins with numerous lymphovenous anastomoses. The pharyngovertebral veins reportedly drain the posterosuperior pharyngeal region and serve as pathways for inflammatory exudate to travel from the pharynx to cervical ligaments, including those around the atlanto-axial joint.

According to the two-hit hypothesis, the first hit is a preexisting cervical ligamentous laxity that is usually seen in the pediatric population, and as the second hit, inflammatory mediators induce a subsequent subluxation of the atlanto-axial joint [5]. Evidence for the twohit hypothesis is the fact that Grisel syndrome is commonly seen in children. The normal range of the anterior atlanto-dens interval is up to $4.5 \mathrm{~mm}$ in children and up to $3 \mathrm{~mm}$ in adults, since children are known to have more ligamentous laxity of the atlanto-axial joint [8]. Although Grisel syndrome is commonly seen in children, several cases have been reported in adults, which indicates that the first hit might not be necessary to the pathogenesis of Grisel syndrome [9].

Although the two-hit hypothesis is widely accepted, there are 4 case reports on Grisel syndrome with initially normal atlanto-axial joint, favoring the single-hit hypothesis. Welinder et al. [10] reported on a 6-year-old boy with Grisel syndrome. The initial CT scan revealed retropharyngeal abscess and a normal alignment of the atlanto-axial joint with an anterior atlanto-dens interval of $1.5 \mathrm{~mm}$. However, sequential CT scans, taken 4 weeks and 2 months after admission, showed progression in the atlanto-axial rotational subluxation with further widening of the anterior atlanto-dens interval of $3 \mathrm{~mm}$ and later 7-8 $\mathrm{mm}$. Kerolus et al. [9] reported on a 71-year-old man with Grisel syndrome following endocarditis. Sequential radiologic studies also revealed a newly developed atlanto-axial rotatory subluxation with widening of the anterior atlanto-dens interval of up to $9 \mathrm{~mm}$. The increase 
in anterior atlanto-dens interval is regarded to represent a dysfunction of the transverse ligament, which is a primary stabilizer of the atlanto-axial joint. The sequence of the atlanto-axial subluxation with a widening of the atlanto-dens interval which was initially in the normal range supports that inflammatory processes could lead to subluxation of the initially normal atlanto-axial joint.

The current case also showed a normal alignment of the atlanto-axial joint with a normal anterior atlanto-dens interval of $1.66 \mathrm{~mm}$ before the onset of torticollis, indicating the absence of a preexisting ligamentous laxity. Serial radiological studies in the current case also suggested that the spread of an inflammatory process that started as retropharyngeal and cervical lymphadenitis resulted in subluxation of the atlanto-axial joint. The limitation of this case is that only static CT was carried out to evaluate the preexisting ligamentous laxity. In this case, an MRI or dynamic CT study was not considered for diagnosis at first since there was no symptom indicating Grisel syndrome initially.

Even with this limitation, the findings of the current case imply that a single hit, such as inflammatory changes in the atlanto-axial joint, could cause Grisel syndrome even without an underlying ligamentous laxity. Therefore, we conclude that Grisel syndrome might be caused by either a single-hit or two-hit mechanism. Nevertheless, more cases are needed to validate this hypothesis.

\section{CONFLICT OF INTEREST}

No potential conflict of interest relevant to this article was reported.

\section{REFERENCES}

1. Karkos PD, Benton J, Leong SC, Mushi E, Sivaji N, As- simakopoulos DA. Grisel's syndrome in otolaryngology: a systematic review. Int J Pediatr Otorhinolaryngol 2007;71:1823-7.

2. Deichmueller CM, Welkoborsky HJ. Grisel's syndrome: a rare complication following "small" operations and infections in the ENT region. Eur Arch Otorhinolaryngol 2010;267:1467-73.

3. Park SH, Park SH, Lee SH. Grisel syndrome: pathophysiological evidence from magnetic resonance imaging findings. Ann Rehabil Med 2013;37:713-6.

4. Wilson MJ, Michele AA, Jacobson EW. Spontaneous dislocation of the atlanto-axial articulation, including a report of a case with quadriplegia. J Bone Joint Surg Am 1940;22:698-707.

5. Battiata AP, Pazos G. Grisel's syndrome: the two-hit hypothesis: a case report and literature review. Ear Nose Throat J 2004;83:553-5.

6. Osiro S, Tiwari KJ, Matusz P, Gielecki J, Tubbs RS, Loukas M. Grisel's syndrome: a comprehensive review with focus on pathogenesis, natural history, and current treatment options. Childs Nerv Syst 2012;28:8215.

7. Parke WW, Rothman RH, Brown MD. The pharyngovertebral veins: an anatomical rationale for Grisel's syndrome. J Bone Joint Surg Am 1984;66:568-74.

8. Jackson $\mathrm{H}$. The diagnosis of minimal atlanto-axial subluxation. Br J Radiol 1950;23:672-4.

9. Kerolus M, Jeans EB, Fontes RB, Deutsch H, Traynelis VC. Atlantoaxial instability of inflammatory origin in adults: case reports, literature review, and rationale for early surgical intervention. Neurosurgery 2015;76:E226-32.

10. Welinder NR, Hoffmann P, Hakansson S. Pathogenesis of non-traumatic atlanto-axial subluxation (Grisel's syndrome). Eur Arch Otorhinolaryngol 1997;254:251-4. 